\title{
Multifocal Pyomyositis
}

\author{
Wg Cdr BM John*, Sqn Ldr SK Patnaik ${ }^{+}$
}

MJAFI 2007; 63 : 191-192

Key Words : Tropical pyomyositis, Multifocal pyomyositis

\section{Introduction}

$\mathrm{P}$ yomyositis is a suppurative infectious disease of the skeletal muscle which is common in tropical countries. The commonest organism implicated is Staphylococcus aureus [1]. Unfamiliarity with the disease, atypical manifestation and a wide range of masquerading conditions often results in a delay in diagnosis. Ultrasound, computed tomography and magnetic resonance imaging (MRI) are useful in establishing a correct diagnosis [2,3]. We present a case of multifocal pyomyositis in a child with involvement of splenius capitis, which has not been reported in literature so far.

\section{Case Report}

A seven year old girl presented with the history of painful swellings over back of neck (left), upper back (right), inner aspect of knee (right) and low grade fever of two weeks duration. The swellings increased in size over the first week and stabilized thereafter. There was no history of trauma or viral illness preceding the onset of complaints. There was no history suggestive of tuberculosis. Examination revealed a well nourished child with low grade fever, tender cystic swellings in the aforementioned sites with mild erythema and

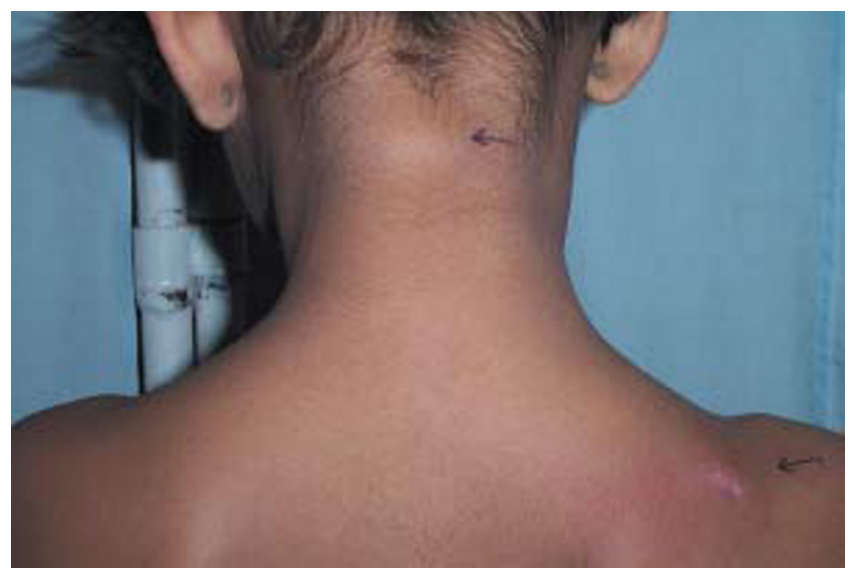

Fig. 1 : Photograph showing swelling over back of neck on left and upper back on right elevation of local temperature (Figs. 1,2). Rest of the clinical examination was unremarkable. The investigations revealed haemoglobin of $10 \mathrm{gm} \%$, total leucocyte count of $11600 /$ cumm, platelet count of 2.2lacs/cumm, peripheral blood smear was normal, erythrocyte sedimentation rate $36 \mathrm{~mm} / 1$ st hour (Westergren), routine urine examination was normal, blood sugar fasting/post prandial was $84 / 112 \mathrm{mg} / \mathrm{dl}$ and blood culture was sterile. The liver function test with enzymes and blood urea/creatinine were normal. Creatine phosphokinase (CPK) $124 \mathrm{U} / \mathrm{L}$, prothrombin time 13sec(test), 12sec (control) activated partial thromboplastin time (APTT) $34 \mathrm{sec}$ (test), $36 \mathrm{sec}$ (control) and human immunodeficiency virus screening was negative. Ultrasonography revealed 17.13 x 8.5 x $12.6 \mathrm{~mm}$ hypoechoic collections within muscles in the neck (splenius capitis), upper back (trapezius) and medial aspect of knee (vastus medialis) respectively. Fine needle aspiration cytology (FNAC) revealed a dense suppurative reaction with sheets of neutrophils in a necrotic background. The pus culture from aspirate grew Staphylococcus aureus sensitive to cloxacillin and gentamicin. Mantoux test and acid fast bacilli culture were negative. The chest radiograph was normal. The child was diagnosed to have multifocal pyomyositis and treated with three weeks of parenteral antibiotics in the form of cloxacillin and gentamicin along with open surgical drainage.

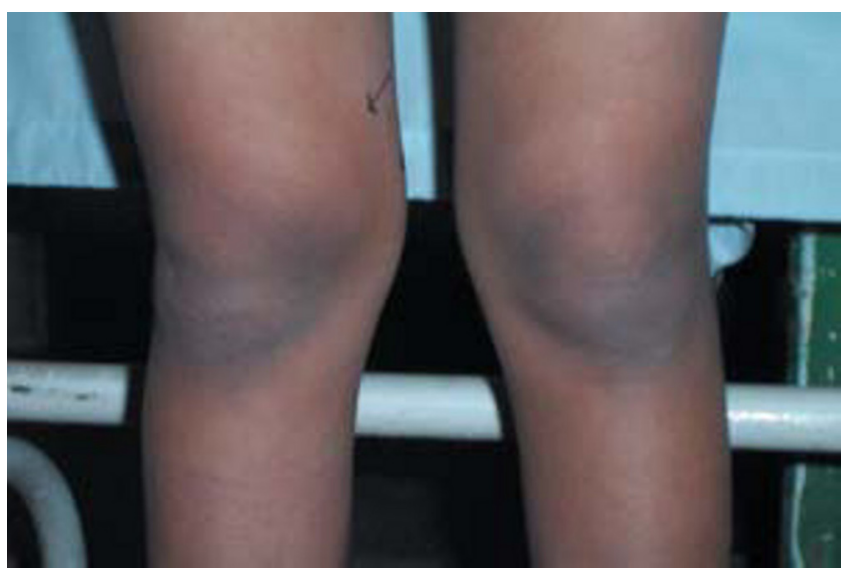

Fig. 2: Swelling over medial aspect of right knee

"Graded Specialist (Paediatrics), ${ }^{+}$Graded Specialist (Paediatrics), 7 Air Force Hospital, Kanpur Cantt. -208004

Received : 05.02.2005; Accepted : 24.04.2006 


\section{Discussion}

Scriba [4], described pyomyositis for the first time in 1885. Pyomyositis is common in tropics and has been widely reported from Asia, Africa and the Caribbean islands, hence the nomenclature of tropical pyomyositis/ myositis tropicans [5]. It has been reported sporadically from our country in paediatric literature [6]. The disease is seen in all age groups, although young males are most susceptible. Maximum incidence is seen at $10-40$ years of age with a male to female ratio of 1.5:1 [5]. The commonest aetiological agent is Staphylococcus aureus. Other organisms like streptococcus (groups A, B, C, G), pneumococcus, neisseria, haemophilus, aeromonas, serratia, yersinia, pseudomonas, klebsiella, escherichia, salmonella, citrobacter, fusobacterium, anaerobes and mycobacterium have also been implicated. Glutei, quadriceps, iliopsoas, gastrocnemius, pectoralis major, serratus anterior, biceps, abdominal and spinal muscles are commonly involved [5]. In the neck region, pyomyositis of sternocleidomastoid only has been described [7].The pathogenesis of pyomyositis is not clear especially as skeletal muscles are usually resistant to microbial invasion. In the immunocompetent host the initiating event is perhaps bacteriological seeding during transient bacteremia against a backdrop of trauma/ vigorous exercise to muscles, underlying viral or parasitic infections or nutritional deficiency [5]. Parasitic diseases have been associated with a switch in the host immune system from a $\mathrm{T}$ helper 1 to a $\mathrm{T}$ helper 2 response that may increase susceptibility to bacterial and fungal infections [8]. Some researchers have proved that the T-cells in patients with tropical pyomyositis are not primed adequately against staphylococcus during the course of infection [5]. The disease is more common in immunocompromised like those with human immunodeficiency virus infection, renal failure or during chemotherapy [5]. The abscesses are mostly solitary but in $12-40 \%$ cases, they may be multifocal [1].

The disease goes through invasive and suppurative stages followed by a stage of complications. Some of the complications are septicaemia, metastatic abscesses, nerve palsy and compartment syndrome $[1,9]$. The differential diagnosis includes pyrexia of unknown origin, muscle contusion, septic arthritis, osteomyelitis, cellulitis, muscle haematoma, deep vein thrombosis, muscle rupture, osteosarcoma of muscle, trichinosis, cysticercosis and polymyositis [1]. Laboratory investigations may reveal anaemia, leucocytosis (shift to the left), raised erythrocyte sedimentation rate and acute phase reactants. Blood cultures may be positive varying from $5 \%-10 \%$ in the tropics to $20 \%-30 \%$ in temperate regions [1]. Serum levels of muscle enzymes such as aldolase and creatine phosphokinase may be slightly raised. Aspiration of pus from the muscle and pus culture is the gold standard for diagnosis [5]. Ultrasound is a simple noninvasive tool for establishing pyomyositis in cases with visible swelling/tenderness. Computed tomography and MRI are useful imaging techniques for early diagnosis [2,3]. Gallium scintigraphy is an extremely sensitive modality for difficult or obscure cases [10].

The management is aggressive with specific parenteral antibiotics covering staphylococcus/other organisms depending on the culture reports along with needle aspiration or open surgical drainage if required [6]. Treatment should usually be continued till wound is clean, the leucocyte count becomes normal, and the patient is afebrile for 7-10 days [5]. A mortality varying from $0.5-2 \%$ underscores the importance of early and appropriate management to prevent complications [1].

\section{Conflicts of Interest}

None identified

\section{References}

1. Christin L, Sarosi GA. Pyomyositis in North America: case reports and review. Clin Infect Dis 1992; 15: 668-77.

2. Trusen A, Beissert M, Schultz G, et al. Ultrasound and MRI features of pyomyositis in children. Eur Radiol 2003; 13: 10505.

3. Gordon BA, Martinez S, Collins AJ. Pyomyositis: characteristics at CT and MR imaging. Radiology 1995; 197 : 279-86.

4. Scriba J. Beitrang zur, Aetiologie der myositis acuta. Deutsche Zeit Chir 1885; 22:497-502.

5. Chauhan S, Jain S, Varma S, Chauhan SS. Tropical pyomyositis (myositis tropicans): current perspective. Postgrad Med J 2004; 80:267-70.

6. Malhotra P, Singh S, Sud A, et al. Tropical pyomyositisexperience of a tertiary care hospital in North West India. J Assoc Physicians India 2000; 48:1057-60.

7. Calduch Broseta JV, Segarra Soria MM, Briceno H, Galvan C.Pyomyositis in sternocleidomastoid muscle. Ann Med Interna 2004; 21:203-4.

8. Lambertucci JR, Rayes AA, Serufo JC, Nobre V. Pyogenic abscesses and parasitic diseases. Rev Inst Med Trop Sao Paulo 2001; 43:67-74.

9. Caparros-Lefebvre D, Strobel M, Ekindi N. Peroneal nerve palsy induced by anterior tibialis pyomyositis. Ann Med Interne 2003; 154:187-9.

10. Schiff RG, Silver L. Tropical pyomyositis: demonstration of extent and distribution of disease by gallium scintigraphy. Clin Nucl Med 1990; 15:542-4. 